\title{
Efficacy and safety of netupitant/palonosetron combination (NEPA) in preventing nausea and vomiting in non-Hodgkin's lymphoma patients undergoing to chemomobilization before autologous stem cell transplantation
}

\author{
Nicola Di Renzo ${ }^{1} \cdot$ Maurizio Musso ${ }^{2} \cdot$ Rosanna Scimè $^{3} \cdot$ Alessandra Cupri $^{4} \cdot$ Tommasina Perrone $^{5} \cdot$ Clara De Risi $^{6}$. \\ Domenico Pastore $^{7}$. Attilio Guarini ${ }^{8} \cdot$ Andrea Mengarelli $^{9}$. Fabio Benedetti ${ }^{10}$. Patrizio Mazza ${ }^{11}$ - Saveria Capria ${ }^{12}$. \\ Patrizia Chiusolo ${ }^{13}$. Luca Cupelli $^{14}$. Vincenzo Federico ${ }^{1}$. Valentina Bozzoli ${ }^{1}$. Anna Rita Messa ${ }^{1}$. Rosella Matera ${ }^{1}$. \\ Davide Seripa ${ }^{1} \cdot$ Paolo Codega $^{15} \cdot$ Erminio Bonizzoni $^{16} \cdot$ Giorgina Specchia $^{5}$
}

Received: 26 February 2021 / Accepted: 9 August 2021 / Published online: 17 September 2021

(c) The Author(s) 2021

\begin{abstract}
Purpose Prevention of chemotherapy-induced nausea and vomiting (CINV) is particularly challenging for patients receiving highly emetogenic preparative regimens before autologous stem cell transplantation (ASCT) due to the daily and continuous emetogenic stimulus of the multiple day chemotherapy. While studies have shown effective prevention of CINV during the conditioning phase with $\mathrm{NK}_{1}$ receptor antagonist $\left(\mathrm{NK}_{1} \mathrm{RA}\right)$-containing regimens, there have been no studies evaluating antiemetic use during chemomobilization prior to ASCT.

Methods This multicenter, open-label, phase IIa study evaluated the efficacy of every-other-day dosing of NEPA administered during chemomobilization in patients with relapsed-refractory aggressive non-Hodgkin's lymphoma. Eighty-one patients participated.

Results Response rates were $77.8 \%$ for complete response (no emesis and no rescue use), $72.8 \%$ for complete control (complete response and no more than mild nausea), $86.4 \%$ for no emesis, and $82.7 \%$ for no rescue use during the overall phase (duration of chemomobilization through $48 \mathrm{~h}$ after). NEPA was well tolerated with no treatment-related adverse events reported.

Conclusion NEPA, administered with a simplified every-other-day schedule, show to be very effective in preventing CINV in patients at high risk of CINV undergoing to chemomobilization of hematopoietic stem cells prior to ASCT.
\end{abstract}

Keywords CINV $\cdot$ Netupitant $\cdot$ Palonosetron $\cdot$ NEPA $\cdot$ ASCT $\cdot$ Multiday chemotherapy

\section{Introduction}

Autologous stem cell transplantation (ASCT) after chemomobilization and stem-cell harvest is widely used as a potential curative strategy to treat non-Hodgkin's lymphoma (NHL). The combination of high-dose chemotherapy with granulocyte colony-stimulating factor (G-CSF) support is a commonly used chemomobilization strategy prior to ASCT [1].

Nicola Di Renzo

direnzo.ematolecce@gmail.com

Extended author information available on the last page of the article
Prevention of chemotherapy-induced nausea and vomiting (CINV) is particularly challenging for patients receiving highly emetogenic preparative regimens before ASCT due to the daily and continuous emetogenic stimulus of the multiple day chemotherapy [2,3]. Evidence-based antiemetic guidelines now recommend co-administration of a combination regimen consisting of a neurokinin-1 $\left(\mathrm{NK}_{1}\right)$ receptor antagonist (RA), 5-hydroxytryptamine-3 (5-HT ${ }_{3}$ ) RA, and dexamethasone (DEX) to prevent CINV in patients who are undergoing conditioning regimens for SCT $[4,5]$. However, the guidelines are remiss in providing specific recommendations on the schedule of these agents in this setting beyond citing aprepitant studies where aprepitant was administered daily during and after 
the preparatory regimen [6-8]. In addition, the guidelines are focused on the conditioning phase prior to ASCT and do not provide any guidance for antiemetic regimens to be used during chemotherapy for mobilization of hematopoietic stem cells.

NEPA is a fixed combination antiemetic uniquely comprised of netupitant, a highly selective $\mathrm{NK}_{1} \mathrm{RA}$ [9] and palonosetron, a second-generation $5 \mathrm{HT}_{3} \mathrm{RA}$, with a higher receptor affinity compared with first generation $5 \mathrm{HT}_{3} \mathrm{RAs}$ [10]. Both molecules have an extended half-life (palonosetron $40 \mathrm{~h}$ and netupitant $90 \mathrm{~h}$ ) relative to other agents in their classes. By simultaneously targeting two critical antiemetic pathways, NEPA offers a simpler more convenient antiemetic with the potential for long-lasting protection from CINV [11]. Oral NEPA plus DEX has shown superiority over oral palonosetron plus DEX for all key efficacy endpoints during the overall ( $0-120 \mathrm{~h}$; 5 days) phase following single-day cisplatin- or anthracycline/cyclophosphamide (AC)-based highly emetogenic chemotherapy (HEC) [12, 13]. However, until recently, trials investigating the efficacy and the safety of NEPA in chemomobilization settings were not available.

The rationale of the current study was to explore the efficacy and the safety of NEPA in preventing CINV in patients with non-Hodgkin's lymphoma (NHL), eligible for ASCT and treated with chemomobilization followed by preparative regimens for ASCT. Results of the conditioning phase of the current study were previously published [14]. The administration of every-other-day NEPA without the addition of DEX was found to be well-tolerated and very effective in controlling both emesis and nausea in patients at high risk of CINV undergoing FEAM/BEAM-based conditioning regimen.

This study differentiates itself from prior trials of aprepitant in this setting. Given the long half-lives of both netupitant and palonosetron, NEPA was administered in the study as an every-other-day regimen, as opposed to daily as in the aprepitant trials. Notably, prior aprepitant studies were performed in combination with DEX as part of the antiemetic regimen [6-8]. However, as DEX exhibits immunosuppressive activity and can result in adverse events, including serious infections in patients undergoing hematopoietic stem cell transplantation, NEPA was administered without DEX in this study, as in previous palonosetron studies in similar settings $[15,16]$.

To our knowledge, this is the first study designed to explore the antiemetic efficacy of an $\mathrm{NK}_{1} \mathrm{RA}$ regimen during the mobilization phase as well as the conditioning phase during which multiple day chemotherapy is administered for mobilizing stem cells prior to ASCT. Prior studies have only evaluated aprepitant/fosaprepitant during the conditioning phase $[17,18]$. The intent of this paper is to present the efficacy and safety of NEPA in preventing CINV associated with the emetogenic multiple drugs administered during the mobilization phase of this study.

\section{Methods}

\section{Study design}

This was a Phase IIa, open-label, non-comparative study with a single-stage Fleming design conducted between January 2016 and February 2018 in 28 Italian centers. Each study center obtained approval from the local institutional review board/ethics committee and all patients provided written informed consent prior to study enrollment.

\section{Patients}

Eligible patients were $\geq 18$ years old with a diagnosis of relapsed/refractory aggressive NHL and eligible for ASCT. Patients were to be appropriate candidates to receive multiple day salvage/mobilization chemotherapy followed by a preparative regimen of BEAM or FEAM prior to ASCT. After enrollment, the patients underwent the mobilization phase. Specific chemomobilization protocols were at the discretion of individual sites and physicians and therefore, consisted of a variety of regimens from $2-5$ days duration. All regimens included chemotherapy agents that are classified as moderately to highly emetogenic according to antiemetic guideline classifications $[4,5]$. Granulocytecolony stimulating factor (G-CSF) support was utilized with or without plerixafor and peripheral collection of autologous CD34 + circulating stem cells by leukapheresis. After hematopoietic stem cell collection and eligibility for ASCT was confirmed, the patients entered the conditioning phase of the study.

\section{Treatment}

During the mobilization phase NEPA was administered every other day of chemotherapy regimen, starting from the first day of chemotherapy administration, with a maximum of 3 total doses given in the case of a 5-day regimen. Use of dexamethasone for antiemetic prophylaxis was not allowed also in patients who did not receive it as a part of chemotherapy regimen, in order to decrease the risk of serious infections in these patients who were already heavily immunosuppressed. Oral metoclopramide (at a maximum dose of $30 \mathrm{mg} /$ day) was used as a rescue antiemetic, if needed.

\section{Assessments}

Nausea and vomiting were self-recorded by patients using a study diary, in which each episode of emesis, any use of 
rescue medication, the maximum grade of nausea according to the Likert scale (none, mild, moderate, and severe), and occurrence of any adverse event were recorded daily from Day 1 of chemomobilization until $48 \mathrm{~h}$ after the last dose of chemotherapy. At the end of the observation period, the patients' global satisfaction with the CINV prophylaxis was also collected by means of a visual analog scale (VAS) from 1 to 10 . Treatment-emergent adverse events (TEAEs) were also monitored and recorded during the study according to the Common Terminology Criteria for Adverse Events version 4.3.

\section{Statistical analysis}

The primary objective of the study was to evaluate the complete response (no emesis and no rescue medication) rate during the overall period of the mobilization phase, defined as from Day 1 of chemotherapy until 2 days after the last dose of chemotherapy. The pre-defined assessment of the efficacy of NEPA for prevention of CINV during the mobilization phase was a secondary objective. Efficacy endpoints for this analysis included complete response (no emesis, no rescue medication), complete control (complete response with a maximum grade of mild nausea), emesis-free (no emesis), rescue-free (no rescue medication), nausea severity, and patient global satisfaction.

Efficacy endpoints were evaluated from Day 1 until $48 \mathrm{~h}$ after the last dose of chemotherapy (i.e., the overall phase) for each patient, as well as, during the acute phase (from Day 1 until last dose of chemotherapy) and delayed phase (from last dose until $48 \mathrm{~h}$ after the last dose). Response was also assessed on each individual day during the overall phase and summarized for the study population. For each endpoint, results were summarized with cumulative incidences with associated two-tailed 95\% exact binomial confidence intervals.

\section{Results}

\section{Patient population}

A total of 82 patients were screened and included in the enrolled population; 81 of these were included in the mobilization analysis (one patient was excluded for undergoing an allogeneic transplantation). Qualitative and quantitative demographic characteristics are summarized in Table 1 and are consistent with the target population of the study. Mobilization included a variety of regimens with or without rituximab. Optimal mobilization was achieved in $97.5 \%$ of patients, with the majority of patients $(90.1 \%)$ undergoing only one apheresis procedure.
Table 1 Summary of patients' characteristics (enrolled population)

\begin{tabular}{|c|c|}
\hline \multicolumn{2}{|l|}{ Age (years) } \\
\hline Mean \pm SD & $54.04 \pm 10.77$ \\
\hline Median (25th-75th) & $56.5(48-62)$ \\
\hline Min-Max & $24-78$ \\
\hline \multicolumn{2}{|l|}{ Gender } \\
\hline Male & $51(62.2 \%)$ \\
\hline Female & $31(37.8 \%)$ \\
\hline \multicolumn{2}{|l|}{ ECOG } \\
\hline 0 & $48(58.5 \%)$ \\
\hline 1 & $29(35.4 \%)$ \\
\hline 2 & $5(6.1 \%)$ \\
\hline \multicolumn{2}{|l|}{ Histology } \\
\hline DLBCL & $77(93.9 \%)$ \\
\hline MCL & $2(2.4 \%)$ \\
\hline PMBCL & $1(1.2 \%)$ \\
\hline PTCL & $2(2.4 \%)$ \\
\hline \multicolumn{2}{|c|}{ Status of disease at study entry } \\
\hline Relapsed & $80(97.6 \%)$ \\
\hline Primary refractory & $2(2.4 \%)$ \\
\hline \multicolumn{2}{|l|}{ No. previous therapy } \\
\hline 1 & $82(100 \%)$ \\
\hline \multicolumn{2}{|c|}{ Duration of mobilization regimen } \\
\hline 2 days & $58(70.7 \%)$ \\
\hline 3 days & $21(25.6 \%)$ \\
\hline 4 days & $1(1.2 \%)$ \\
\hline 5 days & $2(2.4 \%)$ \\
\hline Optimal mobilization & $80(97.5 \%)$ \\
\hline \multicolumn{2}{|l|}{ No. of apheresis } \\
\hline 1 & $74(90.2 \%)$ \\
\hline 2 & $8(9.7 \%)$ \\
\hline \multicolumn{2}{|l|}{ Conditioning regimen } \\
\hline FEAM & $46(56.1 \%)$ \\
\hline BEAM & $23(28.0 \%)$ \\
\hline Melphalan/Mitoxantrone & $1(1.2 \%)$ \\
\hline None & $12(14.6 \%)$ \\
\hline
\end{tabular}

Legend: $D L B C L$, Diffuse Large B-Cell Lymphoma; $M C L$, Mantle Cell Lymphoma; $P M B C L$, Primary Mediastinal B-Cell Lymphoma; $P T C L$, Peripheral T-Cell Lymphoma.

\section{Efficacy}

NEPA was shown to be highly effective in preventing CINV during the mobilization period, particularly for the endpoints of no emesis and no rescue use where overall phase rates were $86.4 \%$ and $82.7 \%$, respectively. The proportions of patients with complete response and complete control during the overall phase were $77.8 \%$ and $72.8 \%$, respectively (Fig. 1). Daily rates for each of these endpoints were consistently above $80 \%$ on each of the individual days during chemomobilization and during the $48 \mathrm{~h}$ after the final dose of chemotherapy. 


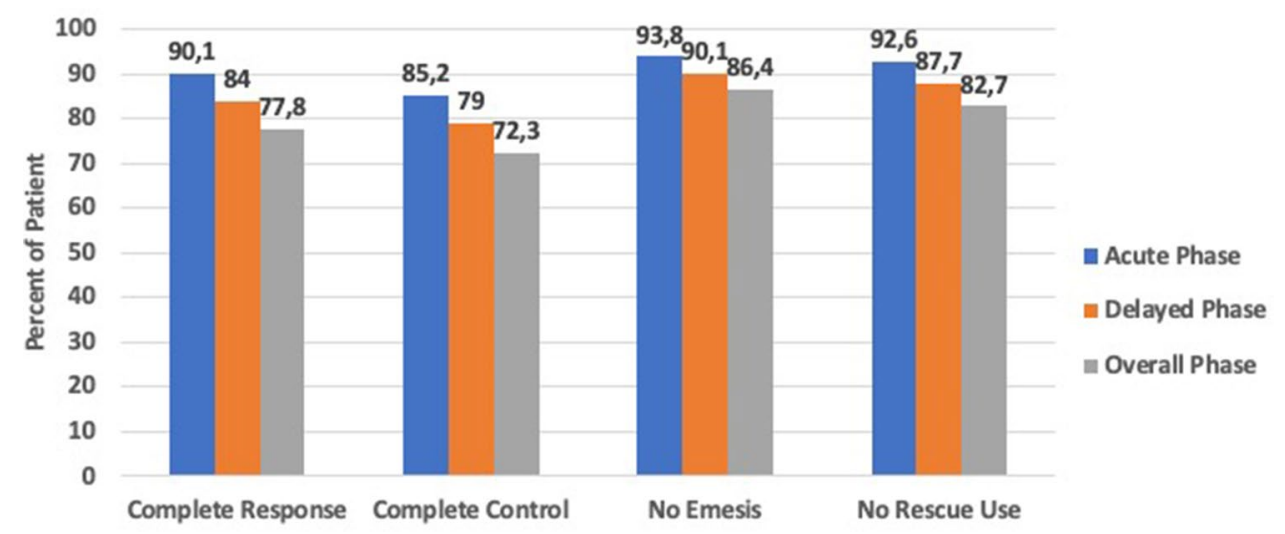

Fig. 1 The histograms show the proportions of patients with complete response (no emesis and no rescue medication), complete control (complete response with no more than mild nausea), no emesis, and no rescue medication during the acute (from Day 1 until last day of chemotherapy), delayed (from last day of chemotherapy until $48 \mathrm{~h}$ after last dose of chemotherapy), and overall (from Day 1 until $48 \mathrm{~h}$ after last dose of chemotherapy) phases during mobilization
Fig. 2 The histograms show the proportions of patients with no, mild, moderate or severe nausea during the acute (from Day 1 until last day of chemotherapy), delayed (from last day of chemotherapy until $48 \mathrm{~h}$ after last dose of chemotherapy), and overall (from Day 1 until $48 \mathrm{~h}$ after last dose of chemotherapy) phases of the mobilization period

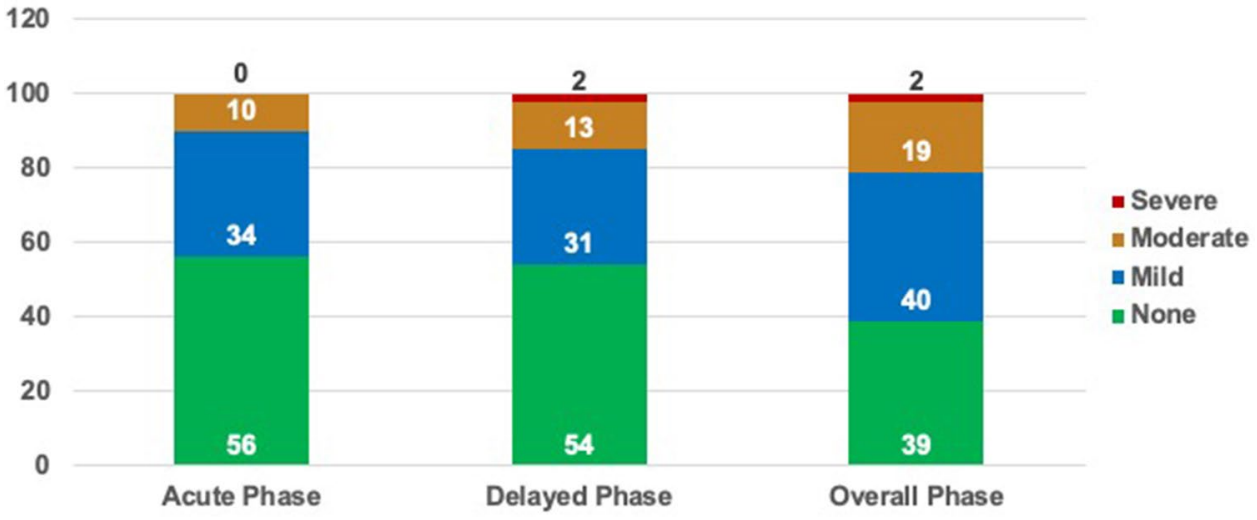

Nausea severity graded on a 4-point Likert scale was also documented by patients. The majority of patients reported no nausea or only mild nausea during the acute, delayed and overall phases (Fig. 2). Severe nausea was only reported by 1 patient $(1.2 \%)$ during the delayed and overall phases. The mean patient global satisfaction with the antiemetic efficacy of NEPA during the mobilization phase was $8.90 \pm 1.56$ out of 10 .

\section{Safety}

NEPA was very well tolerated, with only 10 TEAEs occurring in five $(6.2 \%)$ patients during the mobilization phase of the study. None of these events was evaluated as possibly related to NEPA administration. Two TEAEs (both sepsis) deemed to be unrelated to NEPA were graded as severe, and three unrelated TEAEs (two sepsis and one fatigue) were classified as serious adverse events (SAE).

\section{Discussion}

Despite significant advances in the management of CINV associated with single-day highly and moderately emetogenic chemotherapy, prevention of nausea and vomiting in patients with hematological malignancies has remained a challenge. In this setting, treatments are intense and elicit a persistent emetic stimulus, given that chemotherapy is generally administered repeatedly over multiple consecutive days $[19,20]$. Even with a triple $\mathrm{NK}_{1} \mathrm{RA} / 5-\mathrm{HT}_{3} \mathrm{RA} /$ DEX regimen with components administered daily, response rates in the aprepitant/fosaprepitant studies are inconsistent $[6-8,17]$ and suggest a continued unmet need and opportunity for improved control of CINV, particularly nausea. The results seen in the conditioning phase of our study with NEPA more conveniently administered every-other-day were very encouraging, where a complete response (no emesis and no rescue use) rate of $87 \%$ was shown in the overall phase [14]. In addition, nausea was well controlled, with $93 \%$ of patients experiencing no or 
no more than mild nausea during the overall phase, well above the rates seen in prior aprepitant trials [6-8].

These findings are particularly impressive as this is the first study exploring the efficacy of NEPA in the setting of peripheral progenitor cells mobilization without use of DEX other than included in the chemotherapy regimen. While directly proportional to the total DEX dose and duration administered, the extent of corticosteroid-induced immunosuppression is impossible to predict. In the setting of ASCT, patients undergoing high-dose chemotherapy may develop deep, although transient, immunosuppression which can put the patient's life at risk.

In this case and since some mobilization regimens contain DEX, any additional immunosuppression could lead to an increased risk of infection. This is the reason why the study does not include the administration of DEX for the control of nausea and vomiting induced by regimens that already contain it.

It is noteworthy that the study was conceived in 2015 when there were no guidelines for controlling CINV in this setting of patient. Only recently, NCCN updated guidelines (Version 1.2021) suggesting that DEX should not be added when the anticancer regimen already contains corticosteroids.

Although approximately $70 \%$ of patients in the study received a DEX-containing mobilization regimen, no statistically significant difference was observed in terms of complete responses, complete control, no nausea and rescue medication compared to those who did not receive DEX (Data not shown).

To our knowledge, this is also the first study to explore an antiemetic regimen in preventing CINV associated with multiple day chemotherapy being administered for mobilization of hematopoietic stem cells prior to ASCT. The most common mobilization regimen utilized in this study (DHAP/R-DHAP) is consistent with customary $\mathrm{CT}$ regimens administered and recommended by guidelines for this setting [1]. Consistent with the findings during the conditioning phase, response rates with every-other-day dosing of NEPA during mobilization were high, exceeding $85 \%$ for all efficacy endpoints during chemotherapy administration (acute phase) and ranging from 79 to $90 \%$ across endpoints during the $48 \mathrm{~h}$ following chemotherapy (delayed phase). Emesis control was excellent with $94 \%$ and $90 \%$ of patients experiencing no emesis during the acute and delayed phases, respectively. NEPA effectively controlled nausea as well, with the majority of patients $(\sim 85 \%)$ experiencing no or only mild nausea during the acute and delayed phases.

In conclusion, NEPA was found to be very effective in controlling both emesis and nausea in patients at high risk of CINV undergoing for mobilization of hematopoietic stem cells prior to ASCT. NEPA, as a long-acting highly effective combination antiemetic, simplifies dosing with an every-other-day schedule while also eliminating the use of corticosteroids in these heavily pretreated and immunocompromised patients.

Acknowledgements The authors thank clinical investigators, patients and site personnel who participated in this study. Editorial and medical writing assistance was provided by Jennifer Vanden Burgt, an independent Medical Affairs consultant, Minneapolis, MN and funded by Helsinn Healthcare SA, Lugano, Switzerland.

Author contribution Dr. Nicola Di Renzo and Dr. Paolo Codega contributed equally to the study design, data collection and to write the first draft of manuscript. Dr. Erminio Bonizzoni performed statistical analysis. All authors had significant contributions in revisions of first manuscript, table and figures.

Data availability Yes.

\section{Declarations}

Ethics approval and consent to participate The study was performed in line with the principles of the Declaration of Helsinki.

The study was approved by Local Ethical Committee and registered on AIFA website (Eudra-CT Number 2015-003318-26). Informed consent was obtained from all patients included in the study.

Conflict of interest Dr. Paolo Codega is an employee of Italfarmaco $\mathrm{SpA}$. Other authors have no competing interests to declare.

Consent for publication All authors have approved the final version of this manuscript.

Open Access This article is licensed under a Creative Commons Attribution 4.0 International License, which permits use, sharing, adaptation, distribution and reproduction in any medium or format, as long as you give appropriate credit to the original author(s) and the source, provide a link to the Creative Commons licence, and indicate if changes were made. The images or other third party material in this article are included in the article's Creative Commons licence, unless indicated otherwise in a credit line to the material. If material is not included in the article's Creative Commons licence and your intended use is not permitted by statutory regulation or exceeds the permitted use, you will need to obtain permission directly from the copyright holder. To view a copy of this licence, visit http://creativecommons.org/licenses/by/4.0/.

\section{References}

1. Giralt S, Costa L, Schriber J, Dipersio J, Maziarz R, McCarty J et al (2014) Optimizing autologous stem cell mobilization strategies to improve patient outcomes: consensus guidelines and recommendations. Biol Bone Marrow Transplant 20:295-308

2. Tendas A, Niscola P, Perrotti A, Dentamaro T, de Fabritiis P, Arcese W (2015) Chemotherapy induced nausea and vomiting in bone marrow transplant: the unmet need. Support Care Cancer 23(8):2211

3. Tendas A, Sollazzo F, Bruno A, Cupelli L, Niscola P, Pignatelli $A C$ et al (2012) Obstacles to managing chemotherapy-induced 
nausea and vomiting in high-dose chemotherapy with stem cell transplant. Support Care Cancer 20(5):891-892

4. Hesketh PJ, Kris MG, Basch E, Bohlke K, Barbour SY, ClarkSnow RA et al (2020) Antiemetics: ASCO Guideline Update. J Clin Oncol 38(24):2782-2797

5. Roila F, Molassiotis A, Herrstedt J, Aapro M, Gralla RJ, Bruera E et al (2016) 2019 Updates MASCC and ESMO guideline update for the prevention of chemotherapy- and radiotherapy-induced nausea and vomiting and of nausea and vomiting in advanced cancer patients. Ann Oncol 27:v119-v133

6. Stiff PJ, Fox-Geiman MP, Kiley K, Rychlik K, Parthasarathy M, Fletcher-Gonzalez D et al (2013) Prevention of nausea and vomiting associated with stem cell transplant: results of a prospective, randomized trial of aprepitant used with highly emetogenic preparative regimens. Biol Blood Marrow Transpl 19:49-55

7. Schmitt T, Goldschmidt H, Neben K, Freiberger A, Hüsing J, Gronkowski M et al (2014) Aprepitant, granisetron, and dexamethasone for prevention of chemotherapy-induced nausea and vomiting after high-dose melphalan in autologous transplantation for multiple myeloma: results of a randomized, placebo-controlled phase III trial. J Clin Oncol 32:3413-3420

8. Svanberg A, Birgegård G (2015) Addition of aprepitant (Emend®) to standard antiemetic regimen continued for 7 days after chemotherapy for stem cell transplantation provides significant reduction of vomiting. Oncology 89:31-36

9. Spinelli T, Calcagnile S, Giuliano C, Rossi G, Lanzarotti C, Mair S, Stevens L, Nisbet I (2014) Netupitant PET imaging and ADME studies in humans. J Clin Pharmacol 54(1):97-108. https://doi.org/ 10.1002/jcph. 198

10. Rojas C, Slusher BS (2012) Pharmacological mechanism of 5-HT3 and tachykinin NK-1 receptor antagonism to prevent chemotherapy-induced nausea and vomiting. Eur J Pharmacol 684:1-7

11. Stathis M, Pietra C, Rojas C et al (2012) Inhibition of substance P-mediated responses in NG108-15 cells by netupitant and palonosetron exhibit synergistic effects. Eur J Pharmacol 689(1-3):25-30

12. Hesketh PJ, Rossi G, Rizzi G, Palmas M, Alyasova A, Bondarenko I et al (2014) Efficacy and safety of NEPA, an oral combination of netupitant and palonosetron, for prevention of chemotherapyinduced nausea and vomiting following highly emetogenic chemotherapy: a randomized dose-ranging pivotal study. Ann Oncol 25:1340-1346

13. Aapro M, Rugo H, Rossi G, Rizzi G, Borroni ME, Bondarenko I et al (2014) A randomized phase III study evaluating the efficacy and safety of NEPA, a fixed-dose combination of netupitant and palonosetron, for prevention of chemotherapy-induced nausea and vomiting following moderately emetogenic chemotherapy. Ann Oncol 25:1328-1333
14. Di Renzo N, Musso M, Scimè R, Cupri A, Perrone T, De Risi C et al (2020) Efficacy and safety of multiple doses of NEPA without dexamethasone in preventing nausea and vomiting induced by multiple-day and high-dose chemotherapy in patients with nonHodgkin's lymphoma undergoing autologous hematopoietic stem cell transplantation: a phase IIa, multicenter study. Bone Marrow Transplant. https://doi.org/10.1038/s41409-020-0909-2

15. Di Renzo N, Montanini A, Mannina D, Dondi A, Muci S, Mancuso $S$ et al (2011) Single-dose palonosetron for prevention of chemotherapy-induced nausea and vomiting in patients with aggressive non-Hodgkin's lymphoma receiving moderately emetogenic chemotherapy containing steroids: results of a phase II study from the Gruppo Italiano per lo Studio dei Linfomi (GISL). Support Care Cancer 19(10):1505-1510. https://doi.org/10.1007/ s00520-010-0974-y

16. Choi BS, Borsaru GP, Ballinari G, Voisin D, Di Renzo N (2014) Multicenter phase IV study of palonosetron in the prevention of chemotherapy-induced nausea and vomiting (CINV) in patients with non-Hodgkin lymphomas undergoing repeated cycles of moderately emetogenic chemotherapy. Leuk Lymphoma 55(3):544-550. https://doi.org/10.3109/10428194.2013.813498

17. Tendas A, Marchesi F, Mengarelli A, Annibali O, Tomarchio V, Saltarelli D et al (2019) Prevention of chemotherapy-induced nausea and vomiting after high-dose melphalan and stem cell transplantation: review of the evidence and suggestions. Support Care Cancer 27(3):793-803. https://doi.org/10.1007/ s00520-018-4594-2

18. Clemmons AB, Orr J, Andrick B, Gandhi A, Sportes C, DeRemer D (2018) Randomized, placebo-controlled, phase III trial of fosaprepitant, ondansetron, dexamethasone (FOND) versus FOND plus olanzapine (FOND-O) for the prevention of chemotherapyinduced nausea and vomiting in patients with hematologic malignancies receiving highly emetogenic chemotherapy and hematopoietic cell transplantation regimens: the FOND-O trial. Biol Blood Marrow Transplant 24(10):2065-2071. https://doi.org/10. 1016/j.bbmt.2018.06.005

19. Trigg ME, Inverso DM (2008) Nausea and vomiting with highdose chemotherapy and stem cell rescue therapy: a review of antiemetic regimens. Bone Marrow Transpl 42:501-506

20. Navari RM (2007) Prevention of emesis from multiple-day and high-dose chemotherapy regimens. J Natl Compr Cancer Netw 5:51-59

Publisher's note Springer Nature remains neutral with regard to jurisdictional claims in published maps and institutional affiliations.

\section{Authors and Affiliations}

\section{Nicola Di Renzo ${ }^{1} \cdot$ Maurizio Musso $^{2} \cdot$ Rosanna Scimè $^{3} \cdot$ Alessandra Cupri $^{4} \cdot$ Tommasina Perrone $^{5} \cdot$ Clara De Risi $^{6}$. Domenico Pastore $^{7}$ - Attilio Guarini ${ }^{8} \cdot$ Andrea Mengarelli $^{9}$ - Fabio Benedetti ${ }^{10}$. Patrizio Mazza $^{11}$ - Saveria Capria ${ }^{12}$. Patrizia Chiusolo ${ }^{13}$. Luca Cupelli $^{14}$. Vincenzo Federico ${ }^{1}$. Valentina Bozzoli ${ }^{1}$. Anna Rita Messa ${ }^{1}$. Rosella Matera ${ }^{1}$. Davide Seripa ${ }^{1} \cdot$ Paolo Codega $^{15} \cdot$ Erminio Bonizzoni $^{16} \cdot$ Giorgina Specchia $^{5}$}

1 Hematology and Stem Cell Transplant Unit, "Vito Fazzi" Hospital, Lecce, Italy

2 Hematology and Transplant Unit, Oncology Department La Maddalena, Palermo, Italy

3 UTMO Vito Cervello Hospital, Palermo, Italy
4 Ferrarotto Hospital, Catania, Italy

5 Hematology Unit, Department of Emergency and Organ Transplantation, University of Bari, Bari, Italy

6 Hematology and Transplant Unit, Cardinal Panico Hospital, Tricase, Italy 
7 Hematology Unit, A. Perrino Hospital, Brindisi, Italy

8 Hematology Unit, Giovanni Paolo II IRCCS Cancer Institute Oncology Hospital, Bari, Italy

9 USOD Hematology and Transplant Unit, Regina Elena IFO National Cancer Institute, Roma, Italy

10 Department of Medicine, Section of Hematology and Bone Marrow Transplant Unit, University of Verona, Verona, Italy

11 Department of Hematology-Oncology, Moscati Hospital, Taranto, Italy
12 Department of Translational and Precision Medicine, Hematology Unit, Sapienza University, Policlinico Umberto I, Roma, Italy

13 Hematology Department, Fondazione Policlinico Universitario Agostino Gemelli, Roma, Italy

14 Hematology Unit, Sant' Eugenio Hospital, Roma, Italy

15 Medical Affairs Department, Italfarmaco SpA, Milan, Italy

16 Section of Medical Statistics and Biometry GA Maccacaro, Department of Clinical Science and Community, University of Milan, Milan, Italy 